\title{
Symmetry, Antisymmetry, and Chirality: Use and Misuse of Terminology
}

\author{
Michel Petitjean 1,2 (D) \\ 1 BFA, CNRS UMR 8251, INSERM ERL U1133, Université de Paris, 75013 Paris, France; \\ petitjean.chiral@gmail.com or michel.petitjean@univ-paris-diderot.fr \\ 2 E-pôle de Génoinformatique, CNRS UMR 7592, Institut Jacques Monod, 75013 Paris, France
}

Citation: Petitjean, M. Symmetry, Antisymmetry,and Chirality: Use and Misuse of Terminology. Symmetry 2021, 13, 603. https://doi.org/ $10.3390 /$ sym 13040603

Academic Editor: Alexander Shapovalov

Received: 11 March 2021

Accepted: 2 April 2021

Published: 4 April 2021

Publisher's Note: MDPI stays neutral with regard to jurisdictional claims in published maps and institutional affiliations.

Copyright: (C) 2021 by the author. Licensee MDPI, Basel, Switzerland. This article is an open access article distributed under the terms and conditions of the Creative Commons Attribution (CC BY) license (https:// creativecommons.org/licenses/by/ $4.0 /)$.

\begin{abstract}
We outline the need for rigorous and consensual definitions in the field of symmetry, in particular about chirality. We provide examples of confusing use of such terminology in the mathematical literature and in the physics literature. In particular, we prove that an antisymmetric function is symmetric for a wide class of metrics. It may be either direct-symmetric or achiral or both direct-symmetric and achiral.
\end{abstract}

Keywords: symmetry; antisymmetry; skew-symmetry; chirality

MSC: 00A30; 51F99

\section{Introduction}

Scientific communication, oral or written, is expected to be based on unambiguous concepts. If this is not the case, communication is inefficient: it is a source of confusion, and it is even a potential source of disasters. Students who are taught undefined concepts keep in mind a vague idea of the knowledge transmitted by their teachers. Later, some of these students may become researchers, managers, decision-makers, politicians, or in turn, teachers. All that generates an incredible mess. As everybody knows, disagreements among experts occur frequently. It should be good that these experts have a consensus, at least about the terminology they use to communicate. A famous example is the one of entropy, which generates endless debates between those who consider that thermodynamic entropy is the same as information entropy and those who do not confuse them. Here, we consider the case of symmetry, anti-symmetry, and chirality. This topic has little impact on our everyday lives. It is just an example of the ambiguities arising in the literature.

\section{Symmetry in Mathematics}

It is crucial to understand that mathematical constructs are models of real physical situations and that a mathematical model of symmetry is a simplified image in our mind of some physical situation in which we would like to see symmetry. Here, we focus on the mathematical concept of symmetry. Symmetry has been known for millennia, but its first mathematical presentation seemed to have been due to Weyl [1]. Unfortunately, while Weyl gave a pertinent presentation of the role of groups in symmetry, he did not supply a general definition of symmetry. This can be explained by the fact that several definitions of symmetry exist, depending on which area of mathematics is targeted. Fortunately, the concept underlying most of these definitions is the same, and these latter were unified into a single one [2]. This common definition is the tool we use in the next sections to discuss anti-symmetry and chirality. We summarize this below. 
According to [2]:

- An object is a function having its input argument in a metric space $A$.

We denote by $f$ this function and by $B$ the space of the values returned by $f$.

- An object $f$ is symmetric if a non-identity isometry of $A$ leaving the object invariant exists.

The preserved distance function is denoted $\delta$.

This definition of symmetry works in many situations, such as for geometric figures with or without colors, symmetric functions, matrices, probability distributions, graphs, character strings, etc. It can be used to modelize the many cases of symmetry encountered in the arts, such as paintings, architecture, etc.

Looking at the axioms defining a metric [3], it is observed that they are not used in our definition of symmetry. Thus, the invariance can be extended in the case where the metric is not a true one, such as when $A$ is the Minkowski spacetime [4] (in this case, physicists call $f$ a field) and when $A$ is a quadratic space [5].

We insist that mathematical symmetry is a modeling tool and that it can be used as desired by the experimentalist, so that it may happen that one practical symmetry situation may be described by several mathematical models.

For example, we look at the graph of the even function $f_{1}(x)$ represented in Figure 1. We set $A=\mathbb{R}$ and $B=\mathbb{R}$, and $\delta$ is set as the absolute value of the difference between two real numbers of $A=\mathbb{R}$. Visibly, $\delta$ is preserved by reflection across the origin, that is by the isometry mapping an element $x$ of $A=\mathbb{R}$ to its opposite $-x$, so $f_{1}$ is symmetric.

However, we do not retrieve any invariance for the odd function $f_{2}(x)$ because $f_{2}(-x)=-f_{2}(x)$, so that $f_{2}$ is not invariant for $\delta$ (there is a change of sign). However, as suggested by Figure 1, we would like to recognize that an odd function is symmetric. A way to do that is to notice that the graph of $f_{2}$ is invariant to a rotation of angle $\pi$ in $A \times B=\mathbb{R}^{2}$, but in general, $B$ differs from $A$ (for example, the determinant of a three by three real matrix is an odd function). Then, defining the opposite of an element of $B$ requires that $B$ be at least a group (the inverse of an element is undefined in the case of a monoid). However, endowing $B$ with any mathematical structure would decrease the degree of generality of the symmetry definition.

Indeed, both even functions and odd functions can be recognized as symmetric ones if we consider their graphs as follows. We set $A=\mathbb{R}^{2}$ and $B=\{0,1\}$, and $\delta$ is set to the Euclidean norm of the difference between two points of the real plane. Denoting by $x$ and $y$ the coordinates of a point $z$ of $\mathbb{R}^{2}$, we consider the functions $g_{1}(z)=\mathbf{1}_{y=x^{2}}$ and $g_{2}(z)=\mathbf{1}_{y=x^{3}}$, where 1 denotes the indicator function. It is recalled that, given a set $S, 1_{S}(z)$ returns one when $z \in S$ and zero elsewhere (here, $z=(x, y)$ is a point of $\mathbb{R}^{2}$, and $S$ is a domain of $\mathbb{R}^{2}$ ). We immediately see that $g_{1}$ is invariant by reflection in $\mathbb{R}^{2}$ through the line $x=0$, and we immediately see that $g_{2}$ is invariant by reflection in $\mathbb{R}^{2}$ through the origin.

Both cases of symmetry in Figure 1 were retrieved, but in the case of the even function $f_{1}$, symmetry was retrieved through two ways of modeling symmetry. That exemplifies why symmetry is a modeling tool and that dealing with symmetry without specifying the sets $A$ and $B$, the function $f$, and for which metric $f$ is invariant is a source of ambiguities and contradictions.
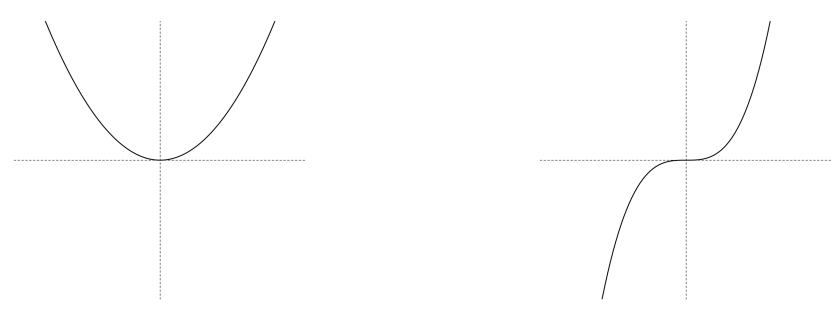

Figure 1. Two simple cases of symmetry. On the left, the graph of the even function $f_{1}: f_{1}(x)=x^{2}$. On the right, the graph of the odd function $f_{2}: f_{2}(x)=x^{3}$. 


\section{Antisymmetry}

Antisymmetry usually refers to relations, functions, and matrices. We see in this section how it relates to the general definition of symmetry. The relations between antisymmetry and chirality are considered in Section 4 . They are used to explain how misuses of terminology can arise.

\subsection{Symmetric and Antisymmetric Relations}

According to [6], a relation $\mathcal{R}$ on a given set is symmetric if whenever $x^{\prime} \mathcal{R} x^{\prime \prime}$, then $x^{\prime \prime} \mathcal{R} x^{\prime}$. Still, according to [6], a relation $\mathcal{R}$ on a given set is antisymmetric if whenever $x^{\prime} \mathcal{R} x^{\prime \prime}$ and $x^{\prime \prime} \mathcal{R} x^{\prime}$, then $x^{\prime}=x^{\prime \prime}$. It follows that a relation $\mathcal{R}$ cannot be both symmetric and antisymmetric, unless $\mathcal{R}$ is restricted to the elements $x$ such that $x \mathcal{R} x$.

In general, a binary relation from a set $A^{\prime}$ to a set $A^{\prime \prime}$ can be modelized with a function $f$ having its input argument in the Cartesian product $A=A^{\prime} \times A^{\prime \prime}$. Then, $f$ is the indicator function of the existence of $x^{\prime} \mathcal{R} x^{\prime \prime}$, where $x^{\prime} \in A^{\prime}$ and $x^{\prime \prime} \in A^{\prime \prime}$. We define $B=\{0,1\}$. Alternatively, $B$ could be something like $B=\{$ false, true $\}$, so that $f$ returns the first element of $B$ when the relation from $x^{\prime}$ to $x^{\prime \prime}$ does not exist, and $f$ returns the second element of $B$ when it exists. This approach can be extended to ternary relations, quaternary relations, etc.

To deal with antisymmetry, we must have $A^{\prime}=A^{\prime \prime}$. For clarity, we consider the case where $A^{\prime}\left(\right.$ or $\left.A^{\prime \prime}\right)$ is of finite cardinality $n$. This set contains the $n$ nodes of the graph of the relation, in the sense of graph theory. In the case of a more general graph, $B$ could be such that $f$ returns the weight of an edge, a color or a tuple of colors, or any attribute of an edge. Here, we consider only the case where an edge exists or not, that is $f$ is the indicator function of the existence of an edge. Each node is allowed to bear a loop.

The metric $\delta$ is the indicator function $\mathbf{1}_{x^{\prime} \neq x^{\prime \prime}}$, which returns the value of zero if and only if the nodes $x^{\prime}$ and $x^{\prime \prime}$ cannot be distinguished. To see why this metric is consistent, we observe that the $n$ ! ways to renumber the nodes leave the graph invariant. The isometries for $\delta$ are the $n$ ! permutations of the nodes, that is there are $n$ ! graph automorphisms. We recall that the set of automorphisms of $A^{\prime}$ (or $A^{\prime \prime}$ ) is a group for the composition of bijections of $A^{\prime}$ onto $A^{\prime}$, called the automorphism group of $A^{\prime}$ or the symmetric group of $A^{\prime}$ (this is also true for an infinite cardinality set $A^{\prime}$ ). The graph is symmetric if at least one non-identity automorphism leaving $f$ invariant exists. This approach can be extended to the case of labeled nodes, that is where the nodes receive weights, or colors, or other attributes. In this case, the number of isometries can be less than $n$ !.

In the case of non-labeled nodes, $\mathcal{R}$ is antisymmetric if its graph contains at most one directed edge between two nodes. Thus, $f$ is such that, for all distinct two nodes $x^{\prime}$ and $x^{\prime \prime}, f\left(x_{1}\right)=1-f\left(x_{2}\right)$, where $x_{1}=\left(x^{\prime}, x^{\prime \prime}\right)$ and $x_{2}=\left(x^{\prime \prime}, x^{\prime}\right)$. If we would like $\mathcal{R}$ to be both symmetric and antisymmetric, no pair of distinct nodes $x^{\prime}$ and $x^{\prime \prime}$ having an edge can be permuted, because isometries such that $f\left(x_{1}\right)=f\left(x_{2}\right)$ are excluded, due to the antisymmetry of $\mathcal{R}$. Therefore, to preserve both symmetry and antisymmetry, the nonidentity isometries are restricted to those permuting distinct nodes without edges and to those permuting distinct nodes bearing loops.

The cases of symmetric relations and of antisymmetric relations are clear, and there is no ambiguity with these two concepts.

\subsection{Odd Functions and Antisymmetric Functions}

\subsubsection{Odd Functions}

Odd functions are sometimes viewed in the literature as antisymmetric functions [7]. Odd real functions returning real values are considered at the end of Section 2, and we concluded that they are symmetric (see Figure 1). This result can be generalized.

In what follows, we denote by $\bar{x}$ the inverse of an element $x$ of a group, whichever group operation is considered. For example, when the group operation is an addition, $\bar{x}$ denotes the opposite of $x$, which is $-x$.

We consider two sets $G^{\prime}$ and $G^{\prime \prime}$ and a function $g$ of a variable $x \in G^{\prime}$, which takes values $g(x) \in G^{\prime \prime}$. We consider an odd function $g$. This means that $g(-x)=-g(x)$ for all 
$x \in G^{\prime}$. The minus sign is a unary operator, which maps an element to its opposite and, in the more general case, to its inverse, in the sense defined by the group operation. The unary minus sign operator is undefined for lighter structures such as monoids or semigroups. Therefore, $G^{\prime}$ must be a group and $G^{\prime \prime}$ another group. It is allowed to have $G^{\prime}=G^{\prime \prime}$, with or without the same group operation. In any case, $g$ is such that $g(\bar{x})=\overline{g(x)}$.

We recall that, for any set $S$, at least one metric can be defined: it is the discrete metric, also called the trivial metric [3]. It is such that the distance between any two elements $x_{1}$ and $x_{2}$ of $S$ is $\mathbf{1}_{x_{1} \neq x_{2}}$. We further denote the discrete metric by $\delta_{1}$, discarding on which set it is defined; it will be clear from the context.

Lemma 1. (a) The discrete metric $\delta_{1}$ is defined on any set. In the case of the Cartesian product $S=S^{\prime} \times S^{\prime \prime}$ of any two sets $S^{\prime}$ and $S^{\prime \prime}, \delta_{1}\left(z_{1}, z_{2}\right)=\mathbf{1}_{z_{1} \neq z_{2}}=\mathbf{1}_{x_{1} \neq x_{2}}+\mathbf{1}_{y_{1} \neq y_{2}}-\mathbf{1}_{x_{1} \neq x_{2}} \mathbf{1}_{y_{1} \neq y_{2}}$ where $z_{1}=\left(x_{1}, y_{1}\right) \in S, z_{2}=\left(x_{2}, y_{2}\right) \in S, x_{1} \in S^{\prime}, y_{1} \in S^{\prime \prime}, x_{2} \in S^{\prime}$, and $y_{2} \in S^{\prime \prime}$.

(b) Several metrics can exist on the Cartesian product of a finite or countable number of metric spaces. The discrete metric is one of them.

Proof. (a) This result is obvious from the definition of the discrete metric.

(b) See Part (a) and [3] (with examples).

Definition 1. Let $G$ be a group. We call an "inverse preserving metric" a metric on $G$ such that, for all $\left(z_{1}, z_{2}\right) \in G^{2}, \delta\left(\overline{z_{1}}, \overline{z_{2}}\right)=\delta\left(z_{1}, z_{2}\right)$. We define $\bar{\Delta}(G)$ as the set of all inverse preserving metrics on $G$.

We notice that $\bar{\Delta}(G)$ is not empty because it contains at least $\delta_{1}$, but not all metrics defined on a group are in $\bar{\Delta}(G)$. We give a counterexample with the finite group $G_{3}=\left\{e, z, z^{2}\right\}$, where $e$ is the neutral element and $\bar{z}=z^{2}$ (so, $\overline{z^{2}}=z$ and $z^{3}=e$ ) and for which the metric $\delta$ such that $\delta(e, z)=2, \delta(e, \bar{z})=4$, and $\delta(z, \bar{z})=5$ is not in $\bar{\Delta}\left(G_{3}\right)$ because $\delta(e, \bar{z}) \neq \delta(e, z)$.

However, a wide class of groups can be endowed with at least one inverse preserving metric (see Definition 1), such as $\mathbb{R}^{d}$ for the addition of vectors.

Theorem 1. Let $G^{\prime}$ be a group containing at least one element distinct from its inverse. An odd function from $G^{\prime}$ to any other group $G^{\prime \prime}$ is symmetric for any metric in $\bar{\Delta}\left(G^{\prime} \times G^{\prime \prime}\right)$.

Proof. We define $A=G^{\prime} \times G^{\prime \prime}, B=\{0,1\}$, and $f(z)=\mathbf{1}_{y=g(x)}$, where $z=(x, y) \in A$. We consider the transformation $T$ mapping any $z=(x, y)$ of $A$ to $T(z)=\bar{z}$, that is $T(z)=(\bar{x}, \bar{y})$. It is easy to see that $T$ is a bijection from $A$ onto $A$. We consider a metric $\delta \in \bar{\Delta}(A)$. Therefore, $\delta\left(T\left(z_{1}\right), T\left(z_{2}\right)\right)=\delta\left(\overline{z_{1}}, \overline{z_{2}}\right)=\delta\left(z_{1}, z_{2}\right)$. Thus, $T$ is an isometry because it preserves $\delta$.

Then, $T$ is not the identity when $A$ contains at least one element, which is not its own inverse, and this is the case because we assumed that $G^{\prime}$ contains at least one element distinct from its inverse.

From Theorem 1, we conclude that calling an odd function antisymmetric is confusing.

\subsubsection{Antisymmetric Functions of Several Variables}

An other case of antisymmetry is when a function of several variables changes sign when two of its arguments are permuted [7]. This case is widely encountered in physics. Speaking about a function of several variables is a common abuse of language, because a function is expected to have only one input argument. For example, if each of the $k$ variables is an element of a set $S$, the unique argument of the function is in the Cartesian product $S^{k}$. Functions of variables that are elements of different sets are handled through an argument in an appropriate Cartesian product. Nevertheless, as the terminology "function of several variables" is unambiguous and widely used, we retain it. 
We consider a function $g$ that is antisymmetric for two of its variables, $x$ and $y$. For clarity, we assume that $g$ is a function of only two variables, but all that follows in this section extends easily to the case where $g$ is a function of more than two variables. Necessarily, the variables $x$ and $y$ must be elements of a common set $S$, because they can be permuted. The antisymmetry means that $g(x, y)=-g(y, x)$. Thus, the function $g$ maps $S^{2}$ to a set $G$, and as noticed in Section 3.2.1, the minus sign means that $G$ must be a group.

Definition 2. Let $S_{1}, S_{2}, \ldots, S_{n}, n \geq 2$, be a family of sets, two of them being identical: $S_{i}=S_{j}$, $1 \leq i \leq n, 1 \leq j \leq n$, and $i \neq j$.

We call a "permutation preserving metric" a metric $\delta$ on the Cartesian product $S=S_{1} \times$ $S_{2} \times \ldots \times S_{n}$, such that the following property stands:

For all two $n$-tuples $\left(\ldots, a_{i}, \ldots, a_{j}, \ldots\right)$ and $\left(\ldots, b_{i}, \ldots, b_{j}, \ldots\right)$ of $S$, where $a_{i} \in S_{i}, b_{i} \in S_{i}, a_{j} \in S_{j}$, $b_{j} \in S_{j}$.

$\delta\left(\left(\ldots, a_{j}, \ldots, a_{i}, \ldots\right),\left(\ldots, b_{j}, \ldots, b_{i}, \ldots\right)\right)=\delta\left(\left(\ldots, a_{i}, \ldots, a_{j}, \ldots\right),\left(\ldots, b_{i}, \ldots, b_{j}, \ldots\right)\right)$.

We define $\widehat{\Delta}(S)$ as the set of all permutation preserving metrics on $S$.

The discrete metric is a permutation preserving metric (see Lemma 1). Not all metrics defined on $S$ are in $\widehat{\Delta}(S)$, but a wide class of permutation preserving product metrics exists.

First, we consider the $n$ metric spaces $\left(S_{k}, d_{k}\right), 1 \leq k \leq n$, and the function $D\left(q_{1}, \ldots, q_{n}\right)$, with $q_{k}=d_{k}\left(a_{k}, b_{k}\right), a_{k} \in S_{k}, b_{k} \in S_{k}, 1 \leq k \leq n$. The function $D$ is a metric on $S$ under the two following conditions [8]:

(1) For all $q \in\left(\left[0, \infty[)^{n}, D(q) \geq 0\right.\right.$, and $D(q)=0 \Leftrightarrow q=0$.

(2) We consider any three elements of $S,\left(a_{1}, \ldots, a_{n}\right),\left(b_{1}, \ldots, b_{n}\right)$, and $\left(c_{1}, \ldots, c_{n}\right)$. Then, we consider the three $n$-tuples of $\left(\left[0, \infty[)^{n}, q^{1}=\left(d_{1}\left(a_{1}, c_{1}\right), \ldots, d_{n}\left(a_{n}, c_{n}\right)\right)\right.\right.$, $q^{2}=\left(d_{1}\left(a_{1}, b_{1}\right), \ldots, d_{n}\left(a_{n}, b_{n}\right)\right)$, and $q^{3}=\left(d_{1}\left(b_{1}, c_{1}\right), \ldots, d_{n}\left(b_{n}, c_{n}\right)\right)$. The function $D$ should be such that, for all $q^{1}, q^{2}$, and $q^{3}$ of $\left(\left[0, \infty[)^{n}\right.\right.$, with $q^{k_{1}} \leq q^{k_{2}}+q^{k_{3}}$, then $D\left(q^{k_{1}}\right) \leq D\left(q^{k_{2}}\right)+D\left(q^{k_{3}}\right)$, where $\left(k_{1}, k_{2}, k_{3}\right)$ is any permutation of $(1,2,3)$.

Usual instances of such a product metric come when the $n$ distance values are viewed as the coordinates of a vector of $\mathbb{R}^{n}$. Endowing $\mathbb{R}^{n}$ with the $p$-norm, $D$ is such that $D\left(\left(a_{1}, \ldots, a_{n}\right),\left(b_{1}, \ldots, b_{n}\right)\right)=\left\|d_{1}\left(a_{1}, b_{1}\right), \ldots, d_{n}\left(a_{n}, b_{n}\right)\right\|_{p}$. Other examples can be found in [3]. Then, the product metric $D$ is permutation preserving when $D\left(\ldots, d\left(a_{i}, b_{i}\right), \ldots, d\left(a_{j}, b_{j}\right), \ldots\right)=D\left(\ldots, d\left(a_{j}, b_{j}\right), \ldots, d\left(a_{i}, b_{i}\right), \ldots\right)$, which means that $D$ is invariant upon the permutation of two members of the $n$-tuple of distance, $d\left(a_{i}, b_{i}\right)$ and $d\left(a_{j}, b_{j}\right)$.

Theorem 2. Let $S$ be any set containing at least two elements, and let $G$ be a group. An antisymmetric function from $S^{2}$ to $G$ is symmetric for any product metric $\delta$ on $S^{2} \times G$ under the conditions that the components $\delta_{S^{2}}$ and $\delta_{G}$ of $\delta$ satisfy: $\delta_{S^{2}} \in \widehat{\Delta}\left(S^{2}\right)$ and $\delta_{G} \in \bar{\Delta}(G)$.

Proof. We define $A=S^{2} \times G, B=\{0,1\}$, and $f(t)=\mathbf{1}_{z=g(x, y)}$, where $t=(x, y, z) \in A$. We consider the transformation $T$ mapping any $t=(x, y, z)$ of $A$ to $T(t)=t^{\prime}=(y, x, \bar{z})$, $t^{\prime} \in A$. It appears that $T$ is a bijection from $A$ onto $A$. The metric $\delta$ is a product metric on $A$, in the sense of [3]. It is such that $\delta\left(T\left(t_{1}\right), T\left(t_{2}\right)\right)=\delta\left(t_{1}, t_{2}\right)$, where $t_{1}=\left(x_{1}, y_{1}, z_{1}\right)$ and $t_{2}=\left(x_{2}, y_{2}, z_{2}\right)$. Therefore, $T$ is an isometry because it preserves $\delta$.

We assumed that $S$ has at least two elements (which can be permuted), so $A$ contains at least two distinct elements, and so, $T$ is not the identity.

Remark 1. Theorem 2 can be extended to antisymmetric functions of $n$ variables, $n>2$, when two of these variables are permuted. The two permuted variables must be elements of the same set, but each of the $n-2$ remaining variables may be an element of any other set. The proof is similar, except that it is more verbose. 


\subsection{Matrices and Tensors}

An antisymmetric square matrix $M$ of $m$ lines and $m$ columns is such that $M$ is equal to the opposite of its transposed $M^{t}$. Each of the $m^{2}$ elements of $M$ must be in the same set $G$. Any set $G$ can be considered, under the condition that it is a group, because the opposite of a matrix element must be defined (see Section 3.2.1 or Section 3.2.2).

We consider the set $S=\{1,2, \ldots, m\}$. A square matrix can be viewed as a function of $S^{2}$ to $G$. Assuming that $m \geq 2$, Theorem 2 applies, and an antisymmetric matrix can be viewed as a symmetric function from $A=S^{2} \times G$ onto $B=\{0,1\}$.

The same way that a matrix is a bi-dimensional array of elements, a tensor can be seen as a higher dimensional array of elements, with additional properties that we do not need here. It follows that any antisymmetric tensor of order $k$ can be viewed as a symmetric function of $k$ variables, and Theorem 2 applies again.

\section{Chirality}

\subsection{Direct and Indirect Isometries}

We saw in Section 3.2 that antisymmetric functions are symmetric (Theorems 1 and 2). However, isometries can be classified as direct or indirect, so that an object can be directsymmetric if it is invariant to a non-identity direct isometry, and it is achiral if it is invariant to an indirect isometry. The terms chiral and chirality were introduced by Lord Kelvin at the end of the 19th Century [9]. Quoting Lord Kelvin: I call any geometrical figure, or group of points, chiral, and say that it has chirality if its image in a plane mirror, ideally realized, cannot be brought to coincide with itself. This definition, which implicitly targets the case of Euclidean spaces, is not mathematical, in a modern sense.

A full mathematical one, based on the classification of isometries as direct or indirect, was given in [10]. This classification and the resulting definition of chirality are recalled here.

- An isometry is direct when it can be written as a product of squared isometries.

- An isometry is indirect when it is not direct.

- An object is achiral when it is invariant to an indirect isometry.

- An object that is not achiral is chiral.

This definition of chirality, which is based on the unifying definition in [2] (see Section 2), recovers the one of Lord Kelvin. However, it works in any metric space, the metric not being required to be a true one, as for the definition of symmetry recalled in Section 2.

A symmetric object may be either direct-symmetric or achiral or both direct-symmetric and achiral. This is why we look below when antisymmetric objects, which we proved to be symmetric for a wide class of metrics (see Section 3.2), are direct-symmetric or achiral.

\subsection{Symmetry of Odd Functions}

An odd function $g$ from a group $G^{\prime}$ to a group $G^{\prime \prime}$ is symmetric (Theorem 1), because there is an isometry $T$ mapping any element $z \in A$, with $A=G^{\prime} \times G^{\prime \prime}$, to its inverse $\bar{z}$ (see Section 3.2.1). This isometry, which preserves any metric of $\bar{\Delta}(A)$, may be direct or indirect.

For example, we consider the case where $G^{\prime}=G^{\prime \prime}=\mathbb{R}$. We have $A=\mathbb{R}^{2}$ and $T(z)=-z, z$ being a point of the real plane $A$. The isometry $T$ is a rotation of angle $\pi$ around the origin. According to the definition of chirality, any rotation is a direct isometry because it can be written as the square of another rotation, but here, rotations are replaced by isometries for any inverse preserving metric (see Definition 1 ) on the real plane $A$, not only for the usual Euclidean metric on $A$. The isometry $T$ is also called a reflection through the origin, but this reflection is not a mirroring operation, although a reflection through a line in the plane is a mirroring operation. In particular, the odd function $f_{2}(x)=x^{3}$ presented in Figure 1 defines a direct-symmetric object (see the end of Section 2).

Similarly, when $G^{\prime}=G^{\prime \prime}=\mathbb{R}^{d}$, the isometry $T$ such that $T(z)=-z, z \in \mathbb{R}^{2 d}$, is a rotation in $\mathbb{R}^{2 d}$ (which can be deduced from the fact that the matrix representing $T$ has its $2 d$ eigenvalues equal to -1$)$. 
Now, we consider the odd function $g$ from $G^{\prime}=\mathbb{R}^{d}$ to $G^{\prime \prime}=\mathbb{R}$, which returns the sum of the coordinates of a vector. The isometry $T$ is such that $T(z)=-z, z \in A$, with $A=\mathbb{R}^{d+1}$. Then, for any inverse preserving metric on $A, T$ is a direct isometry when $d$ is odd, and $T$ in an indirect isometry when $d$ is even. It follows that $g$ is direct-symmetric when $d$ is odd, and $g$ is achiral (that is, indirect-symmetric) when $d$ is even.

The same conclusions occur when we consider the odd function returning the opposite of the sum of the coordinates of a vector of $\mathbb{R}^{d}$.

Remark 2. An object must not be confused with an isometry. This means that the role of a function, when it is viewed as an object, must not be confused with the role of the same function, when it is viewed as an isometry. For example, the function $h$ from $\mathbb{R}$ to $\mathbb{R}$, such that $h(x)=-x$, is indeed a direct-symmetric object, while it is an indirect isometry of $\mathbb{R}$.

\subsection{Functions of Several Variables and Chirality}

We know that antisymmetric functions are symmetric for product metrics satisfying the conditions of Theorem 2. In general, it is hard to conclude about the direct or indirect nature of the symmetry of these antisymmetric functions, even in the case of antisymmetric functions of two variables. Nevertheless, more can be said in some cases of importance. We mainly consider the case of the functions of two variables.

Theorem 3. Let $S$ be any finite set containing at least two elements. An antisymmetric function $g$ of two variables, which maps $S^{2}$ to a finite group $G$, is achiral for any product metric $\delta$ on $S^{2} \times G$ under the conditions that the components $\delta_{S^{2}}$ and $\delta_{G}$ of $\delta$ satisfy: $\delta_{S^{2}} \in \widehat{\Delta}\left(S^{2}\right)$ and $\delta_{G} \in \bar{\Delta}(G)$.

Proof. The set $S$ and the group $G$ are finite, then $A=S^{2} \times G$ is finite, and the group $\Theta$ of isometries of $A$ is finite. From Cayley's theorem [11], the group $\Theta$ is isomorphic with a subgroup of the symmetric group of $\Theta$. This finite subgroup has a permutation representation, which means that it can be represented by a group of permutation matrices.

From Theorem 2, an antisymmetric function from $S^{2}$ to $G$ is symmetric because there is an isometry $T \in \Theta$ mapping any element $t=(x, y, z)$ to $t^{\prime}=(y, x, \bar{z}), t \in A$, $t^{\prime} \in A$. This isometry $T=T_{G} T_{S^{2}}$, where $T_{S^{2}}$ maps $(x, y, z)$ to $(y, x, z)$, and $T_{G}$ maps $(y, x, z)$ to $(y, x, \bar{z})$. Assuming that $S$ has $m$ elements, the permutation representing $T_{S^{2}}$ is the product of $m(m-1) / 2$ transpositions, each transposition permuting the elements $\left(x_{i}, y_{j}, z\right)$ with $\left(y_{j}, x_{i}, z\right), 1 \leq i<j \leq m$. The permutation representing $T_{G}$ is the product of the transpositions permuting the elements $z \in G$ with their inverse $\bar{z}$ when $\bar{z} \neq z$ and of identity permutations in the case where $\bar{z}=z$ (this occurs at least when $z$ is the neutral element of $G)$. These latter transpositions are disjoint, and they are disjoint from the $m(m-1) / 2$ ones due to $T_{S^{2}}$. There is at least one transposition because $m \geq 2$. Overall, none of these transpositions is the product of the squares of permutations, because the permutation matrix associated with a transposition has a determinant of -1 , while a product of squared permutation matrices has a determinant of +1 .

It follows that the overall permutation associated with $T$ cannot be written as a product of squared permutations, discarding whether or not permutations associated with isometries are part of such a product of squares. We deduce that $T$ cannot be written as a product of squared isometries, and so, $g$ is achiral.

In Theorem 3, the case where $S$ has only two elements is of interest. It may erroneously be viewed as a case of chirality, because the two elements of $S$ seem to be mirror images. For example, we may have $S=\{-1,+1\}$, or $S=\{0,1\}$, or $S=\{$ red, green $\}$, or $S=$ $\{$ true, false $\}$, or else.

To see this, we consider an antisymmetric function $g$, such that $g(x, y)=-g(y, x)$, $x \in S, y \in S$. The codomain of $g$ contains $g(x, x), g(y, y), g(x, y)$, and $g(y, x)$. The group $G$ must contain the neutral element $e$ and two other elements, $z$ and its inverse $\bar{z}$, so that $g(x, y)=z$ and $g(y, x)=\bar{z}$. The case where $z=\bar{z}$ has no interest, so it is discarded. Then, the simplest finite group that can be built is $G=G_{3}=\left\{e, z, z^{2}=\bar{z}\right\}$, or any isomorphic one, 
which is such that $g(x, x)=g(y, y)=e$. We consider the discrete metric on $A=S^{2} \times G$. It is a product metric satisfying the conditions of Theorem 3. The isometry that permutes the two elements of $S$ and transforms the element of $G$ to its inverse is an indirect isometry. From Theorem 3, we deduce that $g$ is achiral.

Thus, calling an algebraic structure chiral because it is based on some antisymmetry is confusing. Several algebraic structures were named chiral in the literature [12], such as chiral algebras. These latter require the existence of an antisymmetry property [13]. While the authors are free to call chiral any algebraic structure they like as long as they define it, it remains that it can be confusing for non-experts.

\section{Inconsistency of the Left-Right Classification}

It is common to encounter in the literature the terms right and left to qualify two entities assumed to be mirror images, the former being often associated with a positive value, such as +1 , while the latter is often associated with a negative value, such as +1 . In mathematics, not only the term of mirror images is questionable in some contexts (see Theorem 3 and the end of Section 4.3), but the arbitrary qualification of the two entities as left and right can be misleading, even when mirrors and mirror images are rigorously defined, as occurs in the Euclidean space.

Following a remarkable result of Mezey (see Theorem 10 in [14] and Theorem 1 in [15]), any chiral $n$-point set of $\mathbb{E}^{n}$, the $n$ points being labeled or not, can be deformed continuously into its mirror image without passing through an $n$-achiral intermediate, except in the case of the labeled $n$-dimensional simplex. An example in the plane is given in Figure 2 (for an example in $\mathbb{E}^{n}$, see [16]).

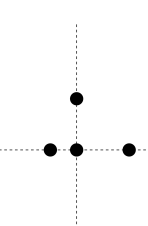

(A)

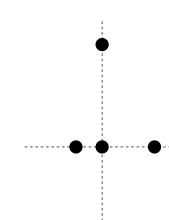

(B)

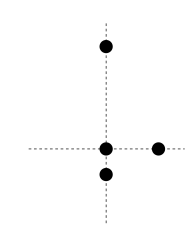

(C)

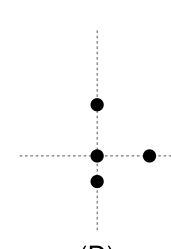

(D)

Figure 2. A four-point set is continuously deformed until it is congruent to its mirror image. (A): $x_{1}=(0,0), x_{2}=(2,0), x_{3}=(0,2), x_{4}=(-1,0) .(\mathbf{B}): x_{1}=(0,0), x_{2}=(2,0), x_{3}=(0,4)$, $x_{4}=(-1,0) .(\mathbf{C}): x_{1}=(0,0), x_{2}=(2,0), x_{3}=(0,4), x_{4}=(0,-1) .(\mathbf{D}): x_{1}=(0,0), x_{2}=(2,0)$, $x_{3}=(0,2), x_{4}=(0,-1)$. From (A) to $(\mathbf{B}): x_{1}=(0,0), x_{2}=(2,0), x_{3}=(0,2+2 \alpha), x_{4}=(-1,0)$, with $\alpha \in\{0,1\}$. From (B) to (C): $x_{1}=(0,0), x_{2}=(2,0), x_{3}=(0,4), x_{4}=(-1+\beta,-\beta)$, with $\beta \in\{0,1\}$. From (C) to (D): $x_{1}=(0,0), x_{2}=(2,0), x_{3}=(0,4-2 \gamma), x_{4}=(0,-1)$, with $\gamma \in\{0,1\}$. The sets (A) and (D) are mirror images through the line in the direction $(1,1)$ and passing by the origin. When going from (A) to (D), all encountered sets are chiral.

It can be concluded that, except for the labeled $n$-dimensional simplex, classifying a chiral $n$-points set of $\mathbb{E}^{n}$ as right or left is meaningless, because the two mirror images are in the same class.

\section{Chirality in Physics}

Many bibliographic searches about chirality in physics leave the readers with the matrix $\gamma^{5}$ (sometimes denoted $\gamma_{5}$, depending on how it is placed in the equations where it appears). This matrix is usually defined as $\gamma^{5}= \pm i \gamma^{0} \gamma^{1} \gamma^{2} \gamma^{3}$, where $i$ is the imaginary number of square -1 and where the four gamma matrices $\gamma^{0}, \gamma^{1}, \gamma^{2}$, and $\gamma^{3}$ are generators of a matrix representation of the Clifford algebra $\mathrm{Cl}_{1,3}(\mathbb{R})$. Originally, these four generating gamma matrices were numbered from 1 to 4 , whence the index 5 for the fifth matrix.

Several reasons can be given by physicists why $\gamma^{5}$ is called a chirality operator (also sometimes called a chiral operator) and why the terms left-chiral and right-chiral are used, but all three terms have to be understood in the context of Dirac spinors, the gamma matrices being expressed in a particular basis called the Weyl basis [17]. 
It seems that the origin of the three terms goes back to 1946 [18], with reference to Kelvin, but without reference to his definition of chirality (see [19] for a small historical survey).

The theory of Dirac spinors is out of the scope of this paper, so we summarize the main equations. A Dirac spinor $\psi$ is an element of $\mathbb{C}^{4}$. Each of its four components is a wavefunction. They are grouped into two pairs, denoted $\phi_{L}$ and $\phi_{R}$, as shown in the equations below, in which $\gamma^{5}$ is a four by four matrix and $\mathbf{1}$ is a two by two identity matrix.

$$
\begin{gathered}
\psi=\psi_{R}+\psi_{L} ; \quad \psi_{R}=\left[\begin{array}{c}
0 \\
\phi_{R}
\end{array}\right] ; \quad \psi_{L}=\left[\begin{array}{c}
\phi_{L} \\
0
\end{array}\right] \\
\gamma^{5}=\left[\begin{array}{cc}
-\mathbf{1} & 0 \\
0 & \mathbf{1}
\end{array}\right] ; \quad \gamma^{5}\left[\psi_{R} \mid \psi_{L}\right]=\left[\psi_{R} \mid-\psi_{L}\right]
\end{gathered}
$$

This means that $\psi_{R}$ and $\psi_{L}$ are the eigenvectors of $\gamma^{5}$, respectively associated with the eigenvalues +1 and -1 , each of these eigenvalues being of multiplicity two.

As mentioned at the end of Section 4, authors are free to call chiral the mathematical structures they like, as long as they define it. However, when the readers are seeking for a definition of chirality in physics, they are mainly redirected to the $\gamma^{5}$ matrix, and then, they may be disappointed because the framework in which $\gamma^{5}$ is defined is very restrictive.

Physicists agree that symmetry is a fundamental concept in physics. Regarding chirality, the situation is different: some physicists consider that chirality is very important, and some other ones consider that it is not a big problem. Anyway, it is unclear how chirality could be defined only from the chirality operator. All that adds to the confusion for non-experts.

\section{Concluding Remarks}

Symmetry seems to be unambiguously understood among the various disciplines where it is used. A proof of that fact is the existence of a unifying definition of symmetry [2]. The situation is different for chirality. In many situations, chirality is invoked, although there is no consensus about what it is (an exception is chemistry, for which chirality is well documented $[20,21])$. This is the case when authors define chirality on the basis of some case of antisymmetry; see Section 4. It is also the case in physics: although physicists seem to agree about what chirality is, it is hard to find a definition suitable for a mathematician.

At the opposite, it happens that chirality is ignored where it should be recognized. This is the case for symmetric probability distributions. While statisticians define unambiguously various types of symmetric multivariate distributions, they do not classify them as direct-symmetric or achiral (or both). However, for example, a symmetric distribution following the univariate normal law is in fact an achiral distribution $[16,22]$. This is so true that a measure of the degree of chirality exists, the chiral index (originally developed for applications in chemistry), which is an asymmetry coefficient devoid of the main drawback of many other asymmetry coefficients: the chiral index is null if and only if the distribution is achiral [16,22-24]. This is not the case of the skewness and its multivariate analogs. For example, the random variable $X$, so that $\operatorname{Prob}(-4)=1 / 3, \operatorname{Prob}(1 / 2)=1$, and $\operatorname{Prob}(5)=1 / 6$, satisfies $E X=0$ and $E X^{3}=0$ [25], while the chiral index if its distribution is $\chi=7 / 60 \approx 0.116667$ [22-24].

The mess surrounding the chirality concept has led to building a general definition of chirality [10], based on the unifying definition of symmetry [2], which is applicable to a vast number of situations. It is expected that it can help readers and future authors willing to publish more about chirality.

Funding: This research received no external funding.

Institutional Review Board Statement: Not applicable.

Informed Consent Statement: Not applicable.

Data Availability Statement: No data were generated during the study. 
Conflicts of Interest: The author declares no conflict of interest.

\section{References}

1. Weyl, H. Symmetry; Princeton University Press: Princeton, NJ, USA, 1952.

2. Petitjean, M. A definition of symmetry. Symmetry Cult. Sci. 2007, 18, 99-119. Available online: https://hal.archives-ouvertes.fr/ hal-01552499 (accessed on 9 March 2021).

3. Deza, M.; Deza, E. Encyclopedia of Distances; Springer: Berlin/Heidelberg, Germany, 2009; Section 1.1 p. 4; Section 1.5 p. 40 ; Section 4.1 p. 83. [CrossRef]

4. Petitjean, M. About chirality in Minkowski spacetime. Symmetry 2019, 11, 1320. [CrossRef]

5. Petitjean, M. Chirality in geometric algebra. Compt. Rend. Math. 2021, submitted.

6. Lipschutz, S.; Lipson, M.L. Schaum's Outline of Theory and Problems of Discrete Mathematics, 3rd ed.; McGraw-Hill: New York, NY, USA, 2007; p. 29.

7. Vince, J. Mathematics for Computer Graphics, 5th ed., Springer: London, UK, 2017; p. 338. [CrossRef]

8. Bernig, A.; Foertsch, T.; Schroeder, V. Non standard metric products. Beitr. Alg. Geom. 2003, 44, 499-510.

9. Thomson, W. The Molecular Tactics of a Crystal; Clarendon Press: Oxford, UK, 1894; Section 22, p. 27.

10. Petitjean, M. Chirality in metric spaces. In memoriam Michel Deza. Optim. Lett. 2020, 14, 329-338. [CrossRef]

11. Robinson, D.J.S. A Course in the Theory of Groups, 2nd ed.; Springer: New York, NY, USA, 1996; p. 36. [CrossRef]

12. Beilinson, A.; Drinfeld, V. Chiral Algebras; AMS Colloquium Publications: Providence, RI, USA, 2004 ; Section 3.

13. Gaitsgory, D. Notes on 2D conformal field theory and string theory. arXiv 1999, arXiv:math/9811061.

14. Mezey, P.G. Rules on chiral and cchiral molecular transformations. J. Math. Chem. 1995, 17, 185-202. [CrossRef]

15. Mezey, P.G. Rules on chiral and cchiral molecular transformations. II. J. Math. Chem. 1995, 18, 133-139. [CrossRef]

16. Petitjean, M. Chirality and symmetry measures: A transdisciplinary review. Entropy 2003, 5, 271-312. [CrossRef]

17. Schwichtenberg, J. Physics from Symmetry, 2nd ed.; Springer: Cham, Switzerland, 2018; Chapters 3.7, 6.3 and 7.4.

18. Eddington, A.S. Fundamental Theory; Cambridge University Press: Cambridge, UK, 1946; p. 111.

19. Petitjean, M. Chirality of Dirac spinors revisited. Symmetry 2020, 12, 616. [CrossRef]

20. Cross, L.C.; Klyne, W. IUPAC rules for the nomenclature of organic chemistry. Section E-stereochemistry (Recommandations 1974). Pure Appl. Chem. 1976, 45, 11-30. [CrossRef]

21. Flapan, E. A Topological Look at Molecular Chirality; Cambridge University Press: New York, NY, USA, 2012. [CrossRef]

22. Petitjean, M. Chiral mixtures. J. Math. Phys. 2002, 43, 4147-4157. [CrossRef]

23. Petitjean, M. Tables of quantiles of the distribution of the empirical chiral index in the case of the uniform law and in the case of the normal law. arXiv 2020, arXiv:2005.09960.

24. Petitjean, M. Extreme asymmetry and chirality. A challenging quantification. Symmetry Cult. Sci. 2020, 31, 439-447. Available online: https: / / hal.archives-ouvertes.fr/hal-03033327 (accessed on 9 March 2021). [CrossRef]

25. Đorić, D.; Nikolić-Đoric, E.; Jevremović, V.; Mališić, J. On measuring skewness and kurtosis. Qual. Quant. 2009, 43, 481-493. [CrossRef] 